\title{
Applied Anatomic Site Study of Palatal Anchorage Implants Using Cone Beam Computed Tomography
}

\author{
Ren-fa Lai ${ }^{1 *}$, Hui Zou ${ }^{1}$, Wei-dong Kong ${ }^{1}$, Wei Lin ${ }^{2}$ \\ ${ }^{1}$ Clinic of Oral \& Maxillofacial Surgery, the First Affiliated Hospital, Jinan University, Guangzhou, China \\ ${ }^{2}$ Academy of Orthodontic/Unit Care Dental Center, Macao, China
}

\section{Abstract}

Aim The purpose of this study was to conduct quantitative research on bone height and bone mineral density of palatal implant sites for implantation, and to provide reference sites for safe and stable palatal implants.

Methodology Three-dimensional reformatting images were reconstructed by cone beam computed tomography $(\mathrm{CBCT})$ in 34 patients, aged 18 to 35 years, using EZ Implant software. Bone height was measured at 20 sites of interest on the palate. Bone mineral density was measured at the 10 sites with the highest implantation rate, classified using K-mean cluster analysis based on bone height and bone mineral density.
Results According to the cluster analysis, 10 sites were classified into three clusters. Significant differences in bone height and bone mineral density were detected between these three clusters $(P<0.05)$. The greatest bone height was obtained in cluster 2 , followed by cluster 1 and cluster 3 . The highest bone mineral density was found in cluster 3 , followed by cluster 1 and cluster 2 .

Conclusion CBCT plays an important role in pre-surgical treatment planning. CBCT is helpful in identifying safe and stable implantation sites for palatal anchorage.

Keywords palatal implant, cone-beam computed tomography, bone height, bone mineral density

Received Feb. 1, 2010; Revision accepted Mar. 26, 2010

\section{Introduction}

Over the last 10 years, palatal implants have become widely used for reliable anchorage in orthodontic treatment. Palatine bone has very irregular morphology and structure. Palatal implantation also involves a risk of damage to the nasal cavity, nasopalatine nerve or maxillary sinus, affecting the implant success rate. It is of practical importance to analyse the bone quantity and quality available in this area. Studies on bone mass and bone mineral density at palatal implant sites before placement are therefore a hot topic in present research. Traditional anchorage techniques, such as wearing headgear and intermaxillary elastic traction, are often unable to meet the clinical demands of orthodontic treatment because the traditional techniques affect appearance, and require compliance of patients, as well as having other unpredictable risks. Palatal implants as an anchorage site have been used in clinical practice for over 10 years, and provide the greatest anchorage force when traditional techniques cannot meet the clinical requirements (Asscherickx et al., 2005; Gracco et al., 2007). Implant anchorage in the palate has numerous advantages compared with conventional anchorage techniques: (1) there is a relatively large bone mass in the median and flanking region palate; (2) there are dense soft tissues on the surface of the hard palate, so compact connective tissues can be formed at the cervical part of the implant; and, (3) a short implant can provide sufficient anchorage. Taken together, palatal implant anchorage has been widely used in clinical practice. However, palatal bone height and bone mineral density differ 
between individuals and between different sites in the same individual. Implantation may damage adjacent structures or result in failed implantation. Gahleitner et al. measured the palatine bone of 32 patients using dental $\mathrm{CT}$, and found that three patients $(9.0 \%)$ could not undergo palatal implant anchorage due to insufficient bone height (Gahleitner et al., 2004). King et al. confirmed that the most suitable region for palatal implantation was $4.0 \mathrm{~mm}$ posterior to and $3.0 \mathrm{~mm}$ lateral to the incisive foramen, but was this region was only suitable for a $3.0-\mathrm{mm}$ long implant (King et al., 2006; Oliveira et al., 2008). Therefore, it is important to assess the bone height and bone mineral density of the palatal implant sites prior to implantation. In 1999, Wehrbein et al. reported that the actual vertical bone height of the anterior and median hard palate was $2 \mathrm{~mm}$ higher than that in lateral cephalometric radiographs (Wehrbein et al., 1999). It is therefore difficult to precisely measure bone height using lateral cephalometric radiographs. The recent introduction of cone beam computed tomography (CBCT) has allowed for the acquistion of high-resolution three-dimensional image, which can be measured using EZ Implant software in axial, coronal and sagittal views, thus avoiding the problems with image clarity and overlapping bone segment.

In this study, we quantitatively analyzed bone height and bone mineral density of the horizontal plate of the palatine bone using CBCT combined with EZ Implant software (Gahleitner et al., 2004). We have also provided references values to identify a safe, stable and reliable palatal implant placement site.

\section{Participants and Methods}

\section{Subjects}

CBCT data from 34 orthodontic patients were selected at the Academy of Orthodontic/Unit Care Center, Macao, China. Inclusion criteria: Chinese; complete maxillary and mandibular dentition; no impacted teeth, no destruction of bone; no tumor in the hard palate; no systemic or metabolic bone disease. The 34 patients included 11 males and 23 females, aged 18-35 (mean $24.2 \pm 1.2$ ) years.

\section{Image taking}

Using CBCT (Classic i-CAT ${ }^{\circledR}$, Imaging Sciences International, USA), cranio-maxillofacial scanning was performed from the frontal to the submental region, $16 \mathrm{~cm}$ in diameter, $13 \mathrm{~cm}$ in height, $120 \mathrm{kV}$ (tube tension), $24 \mathrm{~mA}$ (tube current), $0.4 \mathrm{~mm}$ in scanning layer height, and saved in 14 bit grayscale format. After scanning, data were kept in DICOM format.

\section{Three-dimensional image reconstruction}

The DICOM format data were obtained and analyzed with the EZ Implant computer software (version 1.5, Vatech, Korea). Three-dimensional image reconstruction was performed, with a space resolution of $0.1 \mathrm{~mm}$. Reconstructed computer models were fixed, cut and measured with a measurement tool in the software.

\section{Measurement and calculation}

Measurement of vertical bone height: In the axial plane, a midline sagittal incision was made through the incisive foramen and axia. A coronal incision was made from the posterior border of the incisive foramen, with a spacing of 3.0, 6.0, 9.0 and $12.0 \mathrm{~mm}$. The direction of the incision was simulated as the implanted direction of palatal implant, which was vertical to the contour of the cut point (Figure 1). Coronal planes, at 3.0, 6.0, 9.0 , and $12.0 \mathrm{~mm}$ posterior to the incisive foramen, were labeled P3, P6, P9, P12, respectively (where $\mathrm{P}=\mathrm{Plane}$ ) (Figure 2).

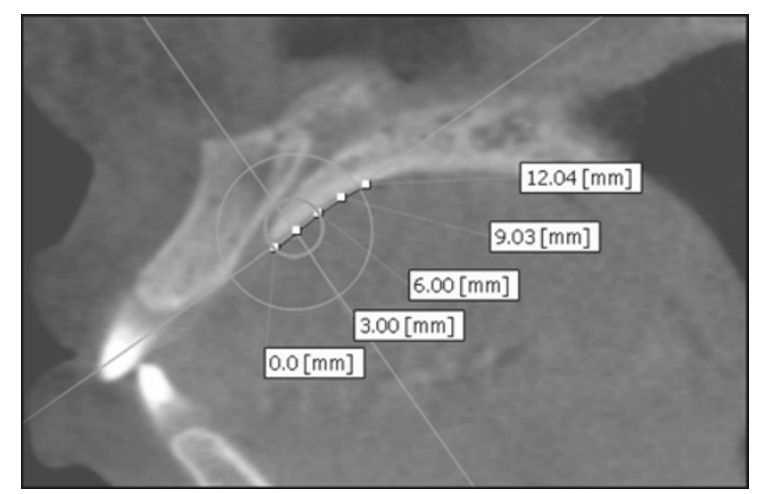

Figure 1 Coronary incision at 3, 6, 9 and $12 \mathrm{~mm}$ posterior to the incisive foramen

Int J Oral Sci, 2(2): 98-104, 2010 - 99 - 


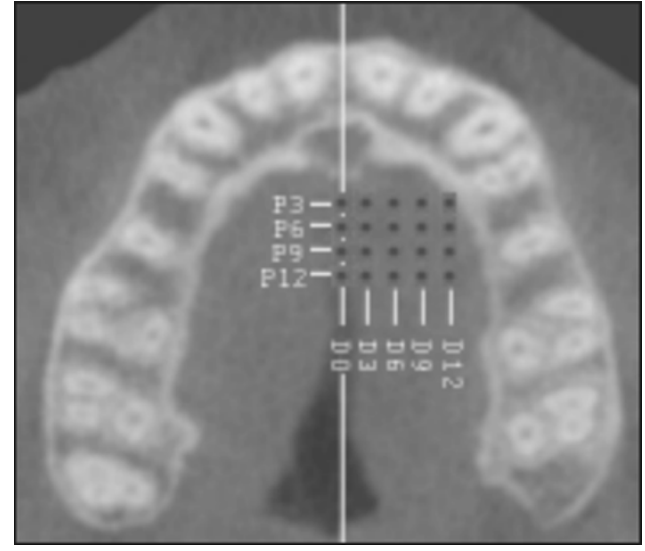

Figure 2 Fixed-point of palate measurement

The height of the hard palatal bone was measured from the left hard palate to $0,3.0,6.0$, 9.0 , and $12.0 \mathrm{~mm}$ from the median line of the palatine bone. The measurement direction was simulated as the implanted direction of the palatal implant, which was vertical to the contour of measurement point (Figures 3-6).

These points at $0,3.0,6.0,9.0$, and $12.0 \mathrm{~mm}$

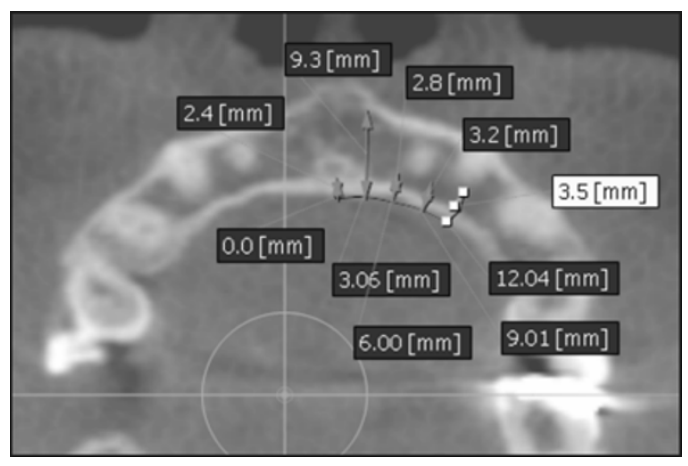

Figure 3 Measurement of vertical bone height on the 3-mm coronal section

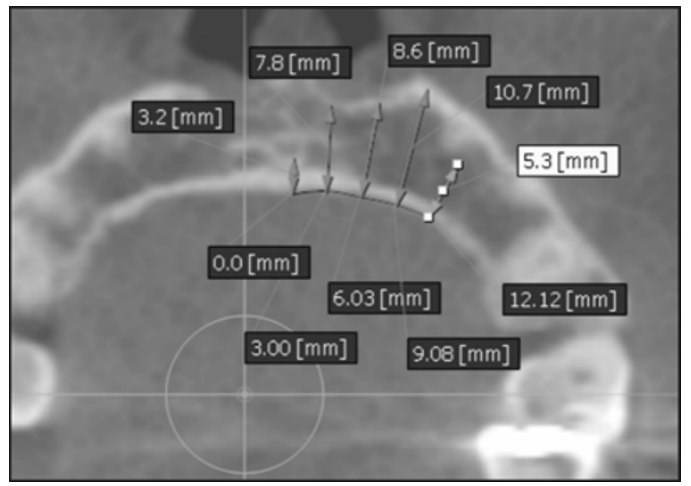

Figure 4 Measurement of vertical bone height on the 6-mm coronal section

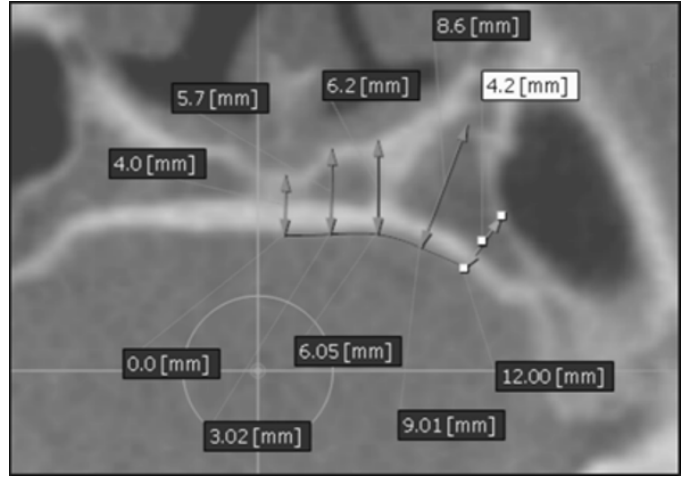

Figure 5 Measurement of vertical bone height on the 9-mm coronal section

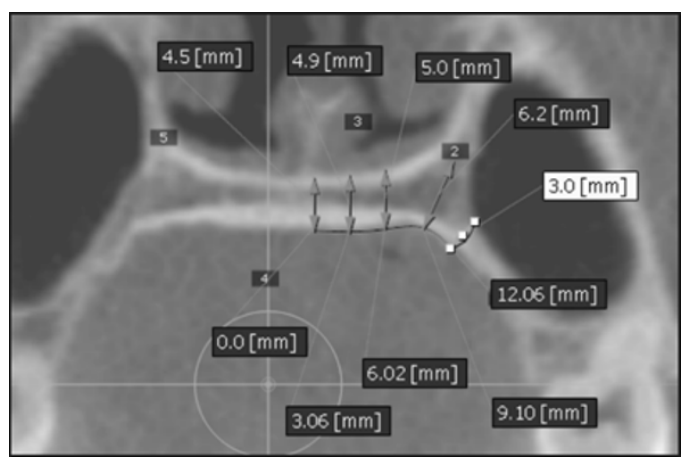

Figure 6 Measurement of vertical bone height on the $12-\mathrm{mm}$ coronal section

from the median line of the palatine bone, were labeled D0, D3, D6, D9 and D12, respectively (where $\mathrm{D}=\mathrm{Distance}$ ) (Figure 2). The 20 measurement points were separately labeled P3D0, P3D3, P3D6, P3D9, P3D12, P6D0-12, P9D0-12, P12D0-12. For example, P3D3 represents the region $3.0 \mathrm{~mm}$ posterior to the incisive foramen and $3.0 \mathrm{~mm}$ from the median line of the palatine bone. The edge of the bone height measurement included the outer layer cortex, the basis nasi, the maxillary sinus floor, the tooth root and lateral wall of the incisive canal of the hard palate bone.

Implantation rate: Considering that the surgical procedure requires a $1.0-\mathrm{mm}$ buffer zone (Kokich, 2004), a site where the vertical bone height is $\geqslant 4.0 \mathrm{~mm}$ was deemed suitable for implantation of a $3.0-\mathrm{mm}$ implant. The implantion rate of a $3.0-\mathrm{mm}$ implant was calculated at 20 sites according to the formula:

$$
\text { Implantation rate }=\frac{\text { Implant number }}{\text { Measurement number }} \times 100 \%
$$


Measurement of bone mineral density: Ten sites with a high implantation rate were selected. Three popular types of cylindrical implants were defined in the software, sized $3.3 \mathrm{~mm}$ diameter $\times 4.0 \mathrm{~mm}$ long; $3.3 \mathrm{~mm}$ diameter $\times 6.0 \mathrm{~mm}$ long; and $3.75 \mathrm{~mm}$ diameter $\times 3.0 \mathrm{~mm}$ long. According to the bone thickness, the most suitable diameter and length of implant was selected for implantation. The distance was $\geqslant 1 \mathrm{~mm}$ between the implant and surrounding structures such as the tooth root of the incisor, the nasal sinus, the maxillary sinus and the incisive canal. The bone mineral density for a region of bone $1 \mathrm{~mm}$ thick surrounding the implant was measured using the EZ Implant software.

\section{Statistical analysis}

Vertical bone height at 20 sites of interest and the bone mineral density at 10 sites with a high implantation rate at the palate are expressed as means. Bone height and bone mineral density of the 10 sites with a high implantation rate were analyzed by K-means clustering with SPSS 13.0 software. Clustering results were analyzed using analysis of variance. The correlation of bone mineral density to bone height was determined (Gahleitner et al., 2004).

\section{Results}

\section{Mean bone height and implantation condition at 20 sites for implantation at the palate}

The thickest part of the palate was detected at P3D6, with a mean bone height of $8.7 \pm 2.7 \mathrm{~mm}$, while the thinnest was measured at P3D0 as $3.6 \pm$ $2.0 \mathrm{~mm}$. The maximal bone height was $15.8 \mathrm{~mm}$, whereas the minimal bone height was $0.8 \mathrm{~mm}$. A total of 10 sites with a high implantation rate were selected, including P3D3, P3D6, P6D0, P6D3, P6D6, P6D9, P9D0, P9D3, P9D9, P12D0 (Table 1).

Table 1 Bone height and implantation condition of 20 palatal implant sites

\begin{tabular}{lcccc}
\hline Site & $\begin{array}{c}\text { Bone height } / \mathrm{mm} \\
(\bar{x} \pm s)\end{array}$ & Maximum $/ \mathrm{mm}$ & Minimum $/ \mathrm{mm}$ & Implantation rate $\%$ \\
\hline P3D0 & $3.6 \pm 2.0$ & 12.9 & 1.7 & 20.6 \\
P3D3 & $5.8 \pm 2.2$ & 10.9 & 2.8 & 76.5 \\
P3D6 & $8.7 \pm 2.7$ & 14.4 & 2.9 & 94.1 \\
P3D9 & $6.7 \pm 3.2$ & 12.4 & 2.3 & 70.6 \\
P3D12 & $3.9 \pm 2.4$ & 12.7 & 1.4 & 35.3 \\
P6D0 & $5.3 \pm 2.0$ & 11.6 & 2.7 & 76.5 \\
P6D3 & $6.4 \pm 2.5$ & 14.4 & 2.8 & 88.2 \\
P6D6 & $7.9 \pm 2.7$ & 14.0 & 3.0 & 91.2 \\
P6D9 & $8.4 \pm 3.4$ & 15.8 & 2.9 & 88.2 \\
P6D12 & $5.7 \pm 2.9$ & 11.2 & 1.8 & 58.8 \\
P9D0 & $5.9 \pm 2.1$ & 13.2 & 3.0 & 88.2 \\
P9D3 & $5.8 \pm 2.6$ & 12.8 & 2.4 & 76.5 \\
P9D6 & $5.8 \pm 2.8$ & 11.5 & 1.6 & 64.7 \\
P9D9 & $6.7 \pm 3.5$ & 14.4 & 1.1 & 79.4 \\
P9D12 & $5.1 \pm 1.5$ & 13.5 & 1.0 & 51.9 \\
P12D0 & $5.1 \pm 1.5$ & 9.4 & 3.2 & 79.4 \\
P12D3 & $4.3 \pm 1.7$ & 8.6 & 1.8 & 58.8 \\
P12D6 & $3.8 \pm 1.8$ & 9.4 & 1.4 & 4.2 \\
P12D9 & $4.2 \pm 2.6$ & 10.2 & 0.8 & \\
P12D12 & $4.8 \pm 3.3$ & 11.4 & & \\
\hline & & & 1.2 & \\
\hline
\end{tabular}




\section{Bone mineral density at 10 sites with a high implantation rate}

The site with the highest mean bone mineral density was P6D0 $(686.0 \pm 134.3 \mathrm{HU})$, whereas the site with the lowest mean mineral density was P9D9 (403.8 $\pm 154.4 \mathrm{HU})$. The highest bone mineral density was $964.0 \mathrm{HU}$, and the lowest bone mineral density was 100.2 HU (Table 2).

Table 2 Bone mineral density (HU) of 10 sites with a high implantation rate for a 3.0-mm long implant

\begin{tabular}{cccc}
\hline Site & $\begin{array}{c}\text { Bone mineral density } \\
\text { /HU }(\bar{x} \pm s)\end{array}$ & Maximum & Minimum \\
\hline P3D3 & $572.0 \pm 138.9$ & 801.4 & 230.0 \\
P3D6 & $414.5 \pm 177.7$ & 800.6 & 120.5 \\
P6D0 & $686.0 \pm 134.3$ & 964.0 & 472.4 \\
P6D3 & $548.6 \pm 159.6$ & 913.1 & 292.1 \\
P6D6 & $426.1 \pm 171.2$ & 850.6 & 178.3 \\
P6D9 & $403.8 \pm 154.4$ & 757.3 & 138.6 \\
P9D0 & $625.9 \pm 144.2$ & 959.1 & 377.1 \\
P9D3 & $510.6 \pm 192.7$ & 923.7 & 183.4 \\
P9D9 & $427.1 \pm 193.8$ & 878.0 & 100.2 \\
P120 & $603.9 \pm 140.2$ & 852.2 & 343.9 \\
\hline
\end{tabular}

\section{Cluster analysis results at $\mathbf{1 0}$ sites with a high implantation rate}

According to the cluster analysis results, the 10 sites could be classified into three clusters. P3D3, P6D3, P9D3 and P9D9 were in class type 1; P3D6, P6D6 and P6D9 were in class type 2; and P6D0, P9D0 and P12D0 were in class type 3 (Table 3 ).
Table 3 Cluster analysis results of 10 sites with a high implantation rate

\begin{tabular}{ccc}
\hline Site & Type & Distance $/ \mathrm{mm}$ \\
\hline P3D3 & 1 & 0.644 \\
P3D6 & 2 & 0.269 \\
P6D0 & 3 & 0.486 \\
P6D3 & 1 & 0.372 \\
P6D6 & 2 & 0.355 \\
P6D9 & 2 & 0.128 \\
P9D0 & 3 & 0.381 \\
P9D3 & 1 & 0.303 \\
P9D9 & 1 & 0.975 \\
P12D0 & 3 & 0.419 \\
\hline
\end{tabular}

\section{Analysis of variance results of the cluster analysis}

As shown in Table 4, there were significant differences in bone height $(F=36.17, P=0.000)$ and bone mineral density $(F=16.52, P=0.002)$ between the three site types. Differences between types were compared using the least significant difference (LSD) method. There were significant differences in bone height in type 2 compared with types 1 and $3(P<0.05)$ and there were significant differences in bone mineral density between all three types $(P<0.05)$. Overall, the rank order for bone height was type $2>$ type 1 and type 3 and that for bone mineral density was type $3>$ type $1>$ type 2 .

\section{Correlation between bone mineral density and bone height}

Pearson's correlation coefficient was $r=-0.874$, $P=0.001$. Bone mineral density was negatively correlated with bone height $(a=0.05)$.

Table 4 Differences in mean bone height and bone mineral density between the three types after cluster analysis

\begin{tabular}{cccccc}
\hline Index & Type & $\begin{array}{c}\text { Mean } \\
(\bar{x} \pm s)\end{array}$ & $F$ & $P$ & Rank order \\
\hline Bone height & 1 & $6.2 \pm 0.5$ & & \\
/mm & 2 & $8.3 \pm 0.4^{\mathrm{a}}$ & 36.17 & 0.001 & Type 2 > type 1, type 3 \\
& 3 & $5.4 \pm 0.4^{\mathrm{b}}$ & & & \\
Bone mineral & 1 & $514.6 \pm 63.6$ & & & Type 3 > type 1 > type 2 \\
density & 2 & $414.8 \pm 11.1^{\mathrm{a}}$ & 16.52 & 0.002 & \\
/HU & 3 & $638.6 \pm 42.5^{\mathrm{ab}}$ & & & \\
\hline
\end{tabular}

${ }^{\mathrm{a}} P<0.05, v s$. type 1 of the same index; ${ }^{\mathrm{b}} P<0.05, v s$. type 2 of the same index. 


\section{Discussion}

The optimal position for implantation depends on the depth, morphology and level of calcification of the hard palatal plate. Insufficient vertical bone height and low bone mineral density are common reasons for failure implantation (Tinsley et al., 1999; Mesa et al., 2008). In this study, the type 2 cluster of sites (P3D6, P6D6, P6D9) had greater bone height, followed by types 1 and 3 . These correspond, respectively to the sites $3.0 \mathrm{~mm}$ posterior to the incisive foramen and $6.0 \mathrm{~mm}$ from the median palate, $6.0 \mathrm{~mm}$ posterior to the incisive foramen, and 6.0 or $9.0 \mathrm{~mm}$ from the median palate. This result differs slightly from those of Bernhart et al. who believed that only the site 6.0-9.0 $\mathrm{mm}$ posterior to the incisive foramen and $3.0-6.0 \mathrm{~mm}$ from the median palate was suitable for implantation (Bernhart et al., 2000). This difference may be due to different races or different age groups in the two studies. Bone mineral density is known to affect the stability of the implant, so the bone mineral density of palatal implant sites should be measured before implantation. We found that type 3 (P6D0, P9D0, P12D0) sites had the greatest bone mineral density at the median palatal suture of the hard palate, followed by types 1 and 2 . The lateral site of the palate has a lower bone mineral density compared with the median palate. Implantation at the median palatal suture appears, therefore, to be the most stable site for implants. However, the bone height of the median palatal suture is lower compared with the lateral site, suggesting that short implants should be used in this region. Uncertainty remains over whether to choose the median palatal suture or the lateral site. Some researchers have shown that the median palatal suture was the center of maxillary growth and development (Bernhart et al., 2000; Schlege et al., 2002; Gahleitner et al., 2004; King et al., 2006). For children and teenagers, implantation should not be conducted at the median palatal suture, but at the lateral region to avoid affecting maxillary growth and development. The anatomic structure of the palatine bone differs between individual and between different sites in the same individual (Lascala et al., 2004; Asscherickx et al., 2005; Kang et al., 2007). Thus, it is necessary to measure the bone height and bone mineral density of the palatal implant sites prior to implantation. Overall, our study reveals that $\mathrm{CBCT}$ is useful to quantitatively analyze bone mineral density and bone height of the palatine bone in order to assess and select suitable palatal implant sites. Results from this study demonstrated that bone mineral density is negatively correlated with bone height. However, the ideal region is not necessarily the region with both the largest bone height and greatest bone mineral density. In the clinic, suitable palatal implant sites can be chosen by assessing the bone height and bone mineral density of the patient's hard palate.

\section{Acknowledgements}

This work was supported by Scientific Research Foundation for Returned Scholars of the Ministry of Education of China (No. 245027), the Science and Technology Planning Program of Guangdong Province (No. 2006B35801004).

\section{References}

Asscherickx K, Hanssens JL, Wehrbein H, Sabzevar MM (2005). Orthodontic anchorage implants inserted in the median palatal suture and normal transverse maxillary growth in growing dogs: a biometric and radiographic study. Angle Orthod, 75(5): 826-831.

Bernhart T, Vollgruber A, Gahleitner A, Dörtbudak O, Haas R (2000). Alternative to the median region of the palate for placement of an orthodontic implant. Clin Oral Implants Res, 11(6): 595-601.

Gahleitner A, Podesser B, Schick S, Watzek G, Imhof H (2004). Dental CT and orthodontic implants: imaging technique and assessment of available bone volume in the hard palate. Eur J Radiol, 51(3): 257-262.

Gracco A, Luca L, Cozzani M, Siciliani G (2007). Assessment of palatal bone thickness in adults with cone beam computerised tomography. Aust Orthod J, 23(2): 109-113.

Kang S, lee SJ, Ahn SJ, Heo MS, Kim TW (2007). Bone thickness of the palate for orthodontic mini-implant anchorage in adults. Am J Orthod Dentofacial Orthop, 131(4 Suppl): S74-S81.

King KS, Lam EW, Faulkner MG, Heo G, Major PW (2006). Predictive factors of vertical bone depth in the 
paramedian palate of adolescents. Angle Orthod, 76(5): $745-751$

Kokich VG (2004). Maxillary lateral incisor implants: planning with the aid of orthodontics. $J$ Oral Maxillofac Surg, 62(9 Suppl 2): 48-56.

Lascala CA, Panella J, Marques MM (2004). Analysis of the accuracy of linear measurements obtained by cone beam computed tomography. Dentomaxillofac Radiol, 33(5): 291-294.

Mesa F, Muñoz R, Noguerol B, de Dios Luna J, O'Valle F (2008). Multivariate study of factors influencing primary dental implant stability. Clin Oral Implants Res, 19(2): 196-200.
Oliveira RC, leles CR, Normanha LM, Lindh C, RibeiroRotta RF (2008). Assessment of trabecular bone density at implant sites on CT images. Oral Surg Oral Med Oral Pathol Oral Radiol Endod, 105(2): 231-238.

Schlegel KA, Kinner F, Schlegel KD (2002). The anatomic basis for palatal implants in orthodontics. Int J Adult Orthodon Orthognath Surg, 17(2): 133-139.

Tinsley D, Watson CJ, Ogden AR (1999). A survey of U.K. centers on implant failures. J Oral Rehabil, 26(1): 14-18.

Wehrbein H, Merz BR, Diedrich P (1999). Palatal bone support for orthodontic implant anchorage - a clinical and radiological study. Eur J Orthod, 21(1): 65-70.

*Corresponding author: Ren-fa Lai

Address: Clinic of Oral \& Maxillofacial Surgery, the First Affiliated Hospital, Jinan University, Guangzhou, Guangdong Province, China

Tel: $862038688109 \quad$ Fax: $862038688000 \quad$ E-mail: tlrf@jnu.edu.cn, Prof.Dr.Lai@163.com 